\title{
Study for Moving Nuclearites and Interstellar Meteoroids using High Sensitivity CMOS Camera
}

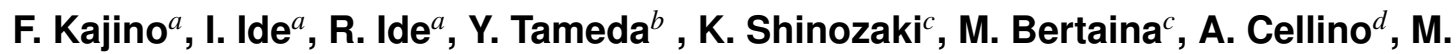 \\ Casolino $^{e, f}$, T. Ebisuzaki ${ }^{e}$, Y. Takizawa ${ }^{e}$, L. W. Piotrowski ${ }^{e}$, H. Sagawa ${ }^{g}$, J. N. \\ Matthews $^{h}$ \\ ${ }^{a}$ Department of Physics, Konan University, Kobe, Hyogo, Japan \\ ${ }^{b}$ Department of Engineering Science, Osaka Electro-Communication University, Neyagawa, \\ Osaka, Japan \\ ${ }^{c}$ Dipartimento di Fisica, Universita' di Torino, Turin, Piedmont, Italy \\ ${ }^{d}$ Osservatorio Astrofisico di Torino, Istituto Nazionale di Astrofisica, Turin, Piedmont, Italy \\ ${ }^{e}$ RIKEN, Wako, Saitama, Japan \\ ${ }^{f}$ Istituto Nazionale di Fisica Nucleare - Sezione di Roma Tor Vergata, Rome, Lazio, Italy \\ ${ }^{g}$ ICRR, University of Tokyo, Kashiwa, Chiba, Japan \\ ${ }^{h}$ Department of Physics and Astronomy, University of Utah, Salt Lake City, Utah, USA \\ E-mail: kajino@konan-u.ac.jp
}

\begin{abstract}
Nuclearites and strange quark matters are hypothetical super-heavy exotic particles and may be important components of the dark matter in our Universe. They may have a typical velocity of about $220 \mathrm{~km} / \mathrm{s}$ (galactic rotation speed), whereas interstellar meteoroids have geocentric velocities larger than $72 \mathrm{~km} / \mathrm{s}$ corresponding to a sum of escape velocity from the solar system and the velocity of the earth around the Sun. We studied the possibility to search for such fast moving particles by using very high sensitivity CMOS cameras. We have observed many meteors using a stereo camera system at the Telescope Array UHECR experiment site in Utah and other places. We estimate the observable mass ranges for the moving nuclearites and the interstellar meteoroids using the observed meteor events. Observable flux limits are also estimated for these mass ranges. Future plans is also reported in this paper.
\end{abstract}

36th International Cosmic Ray Conference -ICRC2019-

July 24th - August 1st, 2019

Madison, WI, U.S.A. 


\section{Introduction}

\subsection{Nuclearite and Strange Quark Matter}

It has been pointed out that strange quark matter (SQM) consisting of aggregates of up, down and strange quarks in roughly equal proportions is more likely to be stable than non-strange quark matter [1]. These nuggets of strange quark matter may be stable for almost any baryon number (A), including values intermediate between those of ordinary nuclei $(\mathrm{A}<263)$ and neutron stars (A $\sim 10^{57}$ ). The above author suggested that nuggets of quark matter could be produced in the firstorder cosmological quark-hadron phase transitions in the early Universe (unlikely) or in processes related to compact stars such as neutron stars or quark stars (more likely). He also suggested the possibility that such nuggets could solve the cosmological dark matter problem.

A. De Rújula and S. L. Glashow used the term 'nuclearite' to indicate large neutral strange quark nugget covered by an electron cloud. Quark nuggets, nuclearites and strangelets are different names used in the literature to indicate so-called strange quark matter. The above authors suggested some experiments to detect nuclearites which are expected to show up as unusual meteor like events, earth-quakes, and etched tracks in old mica, in meteorites and in cosmic-ray detectors [2].

Several experiments have attempted so far to search for such particles in cosmic rays. While some interesting events have been found that are consistent with the predictions for strangelets, none of these have been claimed as real discoveries [3]. Massive nuclearites may pass through the Earth and they may be detectable by seismic signals they generate [2]. D. P. Anderson et al. reported an event that has the properties predicted for the passage of a nugget of strange quark matter through the Earth, although there is no direct confirmation from other phenomenologies [4]. De Rújula et al. calculated the luminosity of the nuclearites when they pass through the atmosphere. The mass-dependent amount of light emitted along the track is quite different with respect to the case of meteors. The emission from nuclearites is expected to occur in a range of heights which is mass-dependent. Moreover, nuclearites may have a typical velocity of $\sim 220 \mathrm{~km} / \mathrm{s}$ near the Earth, whereas the geocentric velocity of the meteoroids bound in the solar system is at most about 72 $\mathrm{km} / \mathrm{s}$. Therefore, they will be able to be distinguished well from the observed data.

\subsection{Meteoroid}

Most meteoroids are small particles belonging to the solar system. The detection of interstellar meteoroids coming from outside our solar system would give us an invaluable scientific information. They are small solid particles in outer space and range in mass roughly from $10^{-19} \mathrm{~kg}$ to $10^{-3}$ $\mathrm{kg}$. They have been detected by space based dust detectors, meteor radars, image intensified video equipment and possibly photographic techniques. Determination of the size distribution, influx rate, dominant directions of arrival and physical and chemical makeup of these meteoroids from interstellar space could significantly constrain models of planetary system formation [5].

At the Earth's orbit, the parabolic or escape velocity with respect to the Sun is about $42 \mathrm{~km} / \mathrm{s}$ and the Earth's orbital speed is about $30 \mathrm{~km} / \mathrm{s}$. Therefore, the geocentric encounter speed of the meteoroids with parabolic orbits could range from about $72 \mathrm{~km} / \mathrm{s}$ to $12 \mathrm{~km} / \mathrm{s}$. It would be expected a heliocentric velocity for a typical interstellar origin meteoroid to be of the order of $47 \mathrm{~km} / \mathrm{s}$ by taking various factors into account [5]. 
We first proposed to study nuclearites and interstellar meteoroids using high sensitivity CMOS camera system in 2017 based on the observational results performed in Jan. 2017 [6].

In this paper, we report some more results from the investigation of the possibility of studying them by using high sensitivity CMOS cameras.

\section{Experimental Apparatus}

Angular velocities of the interstellar meteoroids and nuclearites/SQMs seeing from cameras are generally much larger than those of usual meteoriods. We need, therefore, to use highly sensitive stereo video camera system with high frame rate to define their speed, trajectory, magnitude etc..

We made an assessment of the most adequate camera system for this study. We first studied the topic using high sensitivity CMOS camera system based on the observational results performed in Jan. 2017. We tested 3 types of very high sensitive CMOS cameras, Nikon D5, Canon ME20F-SH color model and Canon ME20F-SH monochrome model at 3 different observation sites during 4 periods in 2017 and 2018. We show their history in Table 1. Rough number of observed meteors for these observation periods are also shown in this table.

Though the ISO sensitivity of Nikon D5 can be set up to $\sim 3$ million equivalent, and that for Canon ME20's up to $\sim 4$ million equivalent, if we set it higher ISO value, the noise level increases more. The ISO value has, therefore, been chosen to set around 204,800 for the observations.

Table 1: Observation history

\begin{tabular}{|c|c|c|c|c|}
\hline $\begin{array}{l}\text { Obs. } \\
\text { period }\end{array}$ & $\begin{array}{l}\text { Obs. } \\
\text { feature }\end{array}$ & $\begin{array}{l}\text { Camera } \\
\text { type }\end{array}$ & $\begin{array}{c}\text { ISO } \\
\text { sensitivity }\end{array}$ & $\begin{array}{l}\text { Obs. } \\
\text { meteors }\end{array}$ \\
\hline $\begin{array}{l}\text { Jan. } \\
2017\end{array}$ & $\begin{array}{l}\text { Stereo obs. } \\
\text { in Hyogo }\end{array}$ & Nikon D5 $\times 2$ & 102,400 & $\sim 80$ \\
\hline $\begin{array}{l}\text { Aug.-Sept. } \\
2017\end{array}$ & $\begin{array}{l}\text { Single camera } \\
\text { at TA site, Utah }\end{array}$ & Nikon D5 & $\begin{array}{c}51,200 \\
-409,600 \\
\end{array}$ & $\sim 300$ \\
\hline $\begin{array}{l}\text { Dec. } \\
2017\end{array}$ & $\begin{array}{c}3 \text { types of cameras } \\
\text { at Ishigaki, Okinawa }\end{array}$ & $\begin{array}{c}\text { Nikon D5 } \\
\text { Canon ME20 mono. \& col. }\end{array}$ & 204,800 & $\sim 300$ \\
\hline $\begin{array}{l}\text { Sept. } \\
2018\end{array}$ & $\begin{array}{c}\text { Stereo obs. using } 3 \\
\text { camaras at TA site, Utah }\end{array}$ & $\begin{array}{c}\text { Nikon D5 } \\
\text { Canon ME20 mono. } \times 2\end{array}$ & 204,800 & $\sim 2,000$ \\
\hline
\end{tabular}

The image sensors of the cameras we used were $35 \mathrm{~mm}$ full-size CMOS sensors. Lens apertures used were $\mathrm{f} / 1.4$ and $\mathrm{f} / 1.2$.

Camera images from each camera are sent to a video capture module through HDMI cable at a rate of 60 or $30 \mathrm{fps}$ with a frame size of $1920 \times 1080$ and then to a PC through a USB cable (Fig. 1). The HDMI (High-Definition Multimedia Interface) is an audio/video interface for transmitting uncompressed video data, and compressed or uncompressed digital audio data from a HDMI-compliant source device. We used AVerMedia HDMI video capture module, CV710, which is compatible with USB 3.0 with a maximum image resolution of $1920 \times 1080$ at a transfer rate of $60 \mathrm{fps}$, and which supports a recording format of uncompressed AVI. 
Though cooled CCD image sensors and raw image format are often used for astronomical observations, we selected to use a camera with a CMOS image sensor and a HDMI interface for image transfer, because we assigned higher priority to high frame rate, high data transfer rate and large sensor size to collect more photons for the present experiment.

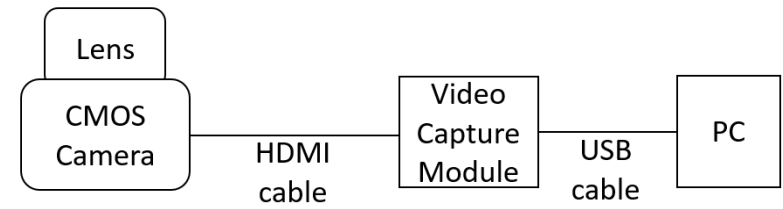

Figure 1: Schematic view of the observation system. Three systems were employed for the observation in Utah, Sept. 2018.

We used a time shifted motion capture software UFOCaptureHD2 [7] among several candidate video softwares [?] to detect and record the meteor events on the PC. Number of head frame before the event trigger start was set to be 30, and that of tail frame after the event trigger end was set to be 30. Number of the frame interval to compare two video images to detect the motion was set to be 1. Scintillation mask option was used to reduce the effect of blinking stars automatically in night sky. This real time function determines the position of long term bright object such as fixed starts in FOV, and mask it with a few pixel around it to avoid a influence of the scintillation caused by atmosphere. This improves the motion detection sensitivity dramatically. There are 3 threshold levels concerning the video image trigger, detection level, detection size and duration of change. The detection level is the brightness difference value between two frames of the same pixel, which is automatically controlled by a detection level noise tracking function.

The detection size is the number of pixels that have changed more than the detection level, which was set to be 3. Minimum number of continuously changed frames by which video trigger should be asserted, was set to be 2 . Triggered events are recorded with uncompressed AVI format in SSD.

\section{Details of Observation in 2018}

As it is important to determine the velocity and trajectory of the interstellar meteoroids and

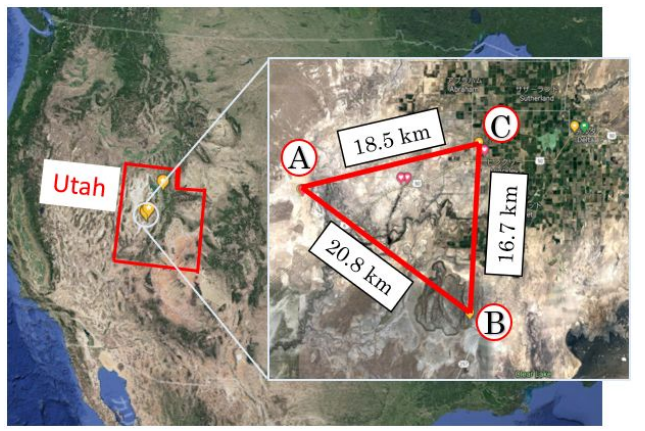

Figure 2: Observation locations in Utah, Sept. 2019 (@)2019 Google).

the nuclearites, to test this concept we set 3 types of camera systems at 3 locations A, B and C separated roughly by $20 \mathrm{~km}$ from each other at the Telescope Array experiment site in Utah in

Table 2: Camera systems used for the observation period in Sept. 2018

\begin{tabular}{|c|c|c|c|c|c|}
\hline System No. & Obs. site & Camera & Focal length & F-number & HDMI FoV \\
\hline No. 1 & A (CLF) & Canon ME20-F mono. & $35 \mathrm{~mm}$ & 1.4 & $54^{\circ} \times 30.4^{\circ}$ \\
\hline No. 2 & B (BRM) & Canon ME20-F mono. & $50 \mathrm{~mm}$ & 1.2 & $40^{\circ} \times 22.5^{\circ}$ \\
\hline No. 3 & C (Hincley) & Nikon D5 & $35 \mathrm{~mm}$ & 1.4 & $52.4^{\circ} \times 29.4^{\circ}$ \\
\hline
\end{tabular}


Sept. 2018. The observation location is shown in Fig. 2 and details of the observation system at this period are shown in Table 2. The frame rate of the camera was chosen to be $29.97 \mathrm{P}$ where $\mathrm{P}$ stands for the progressive mode.

We observed roughly 2000 meteors by summing the events obtained at locations $\mathrm{A}, \mathrm{B}$ and $\mathrm{C}$ in total with the observation during 4 nights in Sept. 2018 period . An example of a composite picture including over 400 meteor events is shown in Fig. 3. We observed the night sky around the direction of Polaris which is shown at the center of the picture.

\section{Analysis}

To obtain various parameters such as the magnitudes at the bright-

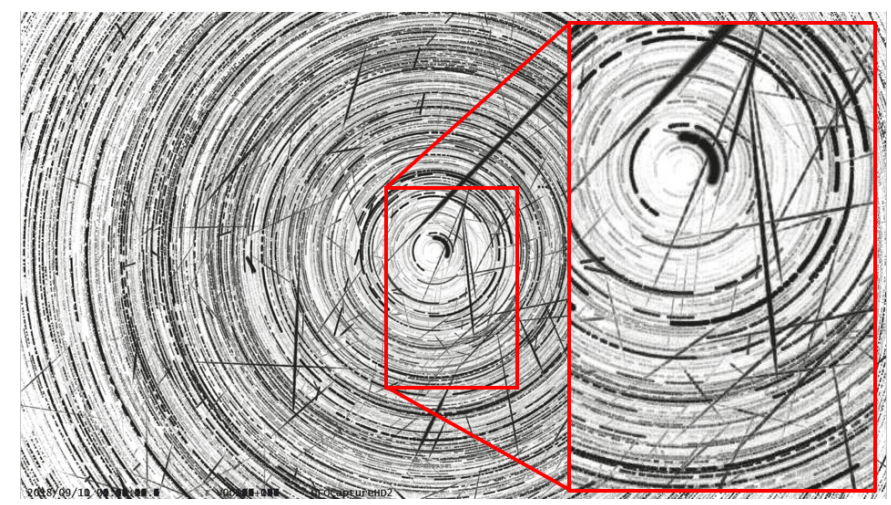

Figure 3: A composite picture including many meteor events, which is taken by the No.2 system at the location B in Utah, Sept. 2018. Black and white colors are inverted for a better visibility. est position along the meteor tracks from the observed meteor video images, the angular velocities of the meteors etc., we used a meteor image analysis software UFOAnalyzerV2 [7] which utilizes a star catalog, SKY2000 Master Catalog Version 4 V/109 [8], containing an extensive compilation of information on almost 300,000 stars brighter than $8.0 \mathrm{mag}$.

\section{Results and Discussion}

An example of meteor event rates in every 10 min as a function of observation time for the 3 systems are shown in Fig. 4. These rates are obtained based on our analysis. The total number of events turns out to be about 500. As the F-number of No. 2 system is smaller than that of No. 1 system, No. 2 system can observe fainter meteors. However, as the field of view (FoV) of the HDMI output of No. 2 is 1.8 times smaller than that of No. 1, their meteor rates became similar as a result.

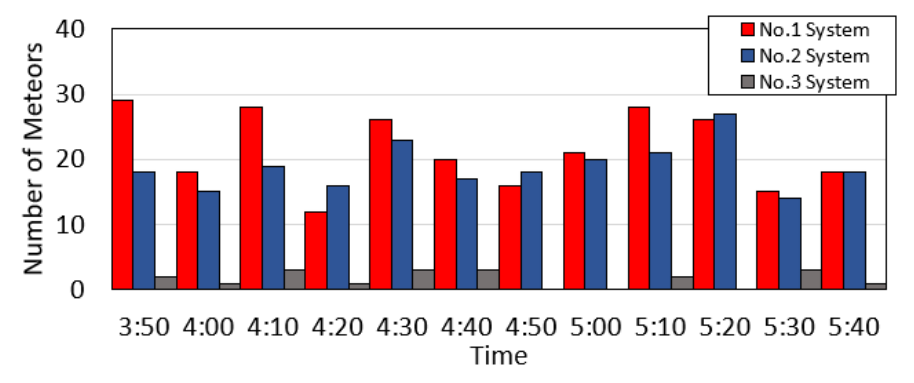

Figure 4: An example of meteor event rates vs. time for 3 camera systems used on Sept. 8, 2018. 
Cumulative event rates of the meteors for No. 1 and No. 2 systems are shown in Fig. 5. Results for various other experiments are also shown in the figure. We could observe meteors with magnitude between 1.5 and 4 by No. 1 system, whereas between 2 and 5 by No. 2 . Based on this figure we can say that our present results about the cumulative meteor rate between observed magnitudes are consistent with other results.

Meteor magnitude as a function of the angular velocity for No.1 and No. 2 systems for each event are plotted in Fig. 6.

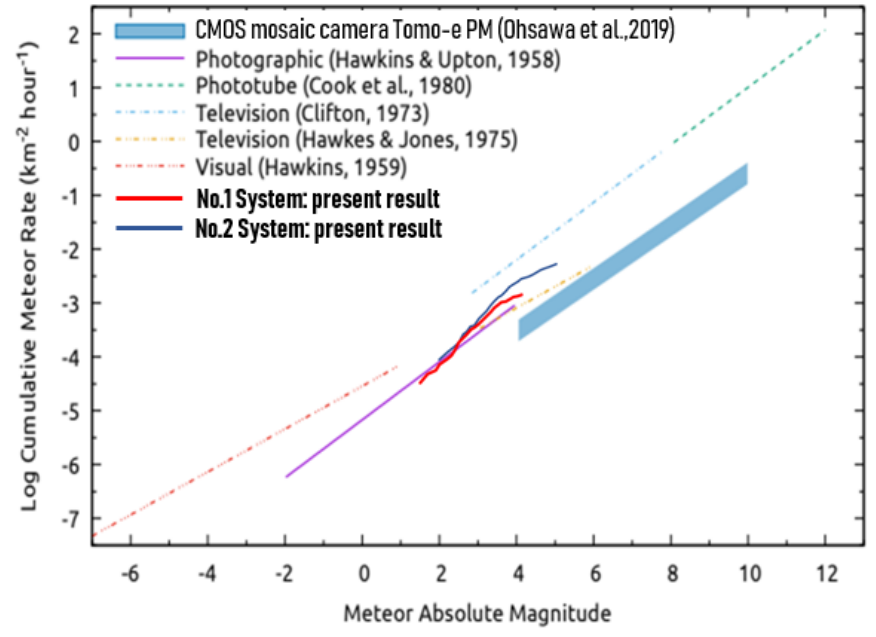

Figure 5: Cumulative meteor rates observed in Utah, Sept. 2019 for No. 1 and No. 2 systems. Results for various other experiments are also shown.
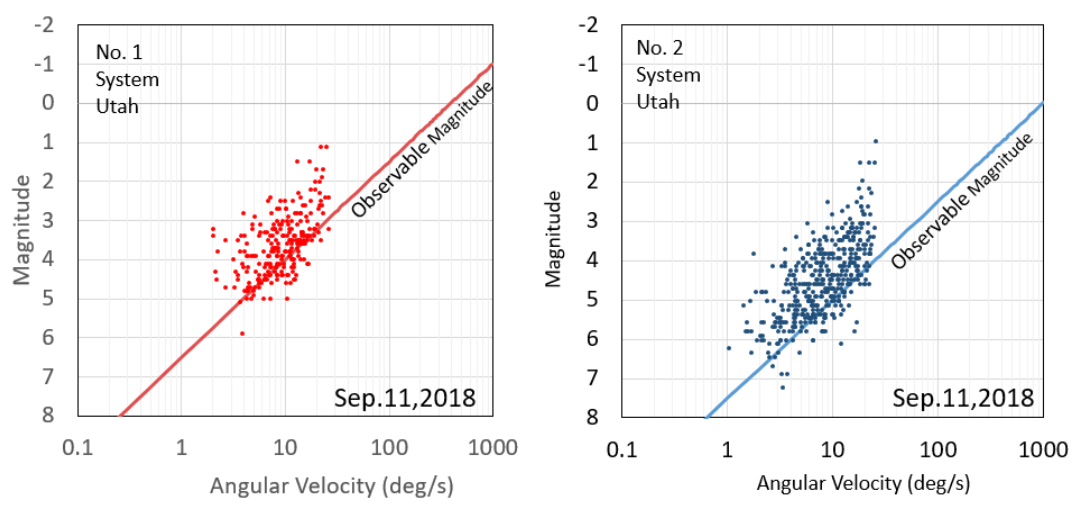

Figure 6: Meteor magnitude vs. angular velocity for No.1 system (left) and No. 2 system (right).

Estimated observable magnitudes as a function of the angular velocities are drawn by solid lines in the figure. The meteors with magnitudes brighter than these lines will be observed with high efficiencies.

Hill et al. suggested that at least high geocentric velocity meteors with masses larger than about $10^{-8} \mathrm{~kg}$ should be observable with current meteor electro-optical technology although there may be observational biases against their detection [9]. They showed the maximum light intensity of meteors as a function of their velocity for various masses of the

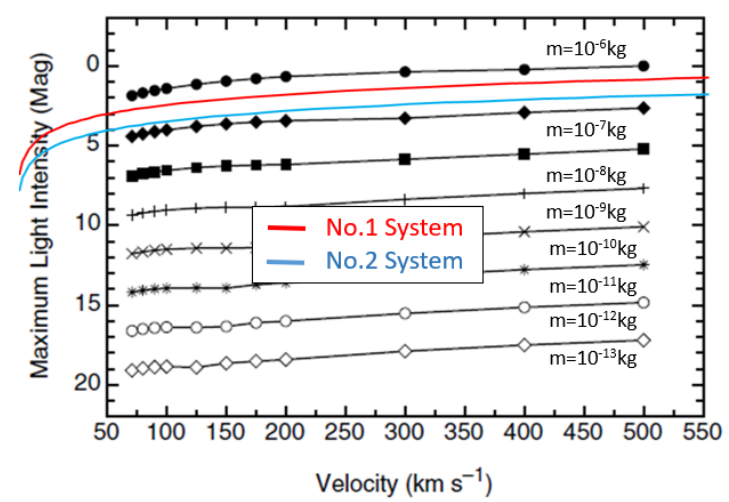

Figure 7: Maximun light intensity of meteors vs. their velocity for various meteoroid masses [9] 
meteoroids (Fig. 7). If the meteor has a velocity sufficiently larger than $72 \mathrm{~km} / \mathrm{s}$, it will be originated by interstellar meteoroids coming from outside our solar system.

Using the lines of the observable magnitude obtained from Fig. 7 by assuming the typical height of the maximum light intensity of the meteor to be $100 \mathrm{~km}$ high from the ground, we have obtained the limits of the observable magnitude as a function of velocity for No. 1 and No. 2 systems, which are drawn in this figure. We can, therefore, obtain the minimum mass limits for the observation of the interstellar meteoroids to be about $4 \times 10^{-7} \mathrm{~kg}$ for No.1 system and $1.5 \times 10^{-7}$ $\mathrm{kg}$ for No. 2 system, respectively. Observable effective area of the system at $100 \mathrm{~km}$ high to the direction of the celestial north pole is obtained by taking into account the following factors. (1) Typical luminous height of the meteors is roughly $100 \mathrm{~km}$. (2) FoV of the observation system.

Expected flux limit for observing interstellar meteoroids, therefore, become about $1.3 \times 10^{-17}$ $\mathrm{m}^{-2} \mathrm{~s}^{-1}$ (No. 1) and $2.9 \times 10^{-17} \mathrm{~m}^{-2} \mathrm{~s}^{-1}$ (No. 2) for the observation period of $1 \mathrm{yr}$ at mass regions larger than $4 \times 10^{-7} \mathrm{~kg}$ (No. 1) and $1.5 \times 10^{-7} \mathrm{~kg}$ (No. 2), respectively, by assuming the efficiency of the observation time to be 0.09 . This limit is drawn in Fig. 8 with many other experiments [10].

Nuclearites, like meteors, produce visible light as they traverse the atmosphere [2]. Observable mass limit of the nuclearite can be obtained using the observable magnitude lines shown in Fig. 6. Nuclearites essentially produce light at much lower altitude than meteors. We will show expected mass limit and the flux limit in near future using the such observable magnitude for our apparatus.

We are going to construct 4 CMOS camera stations at the TA site in Utah to obtain velocities and 3 dimensional trajectories of meteor events for the search for nuclearites and the observation of interstellar

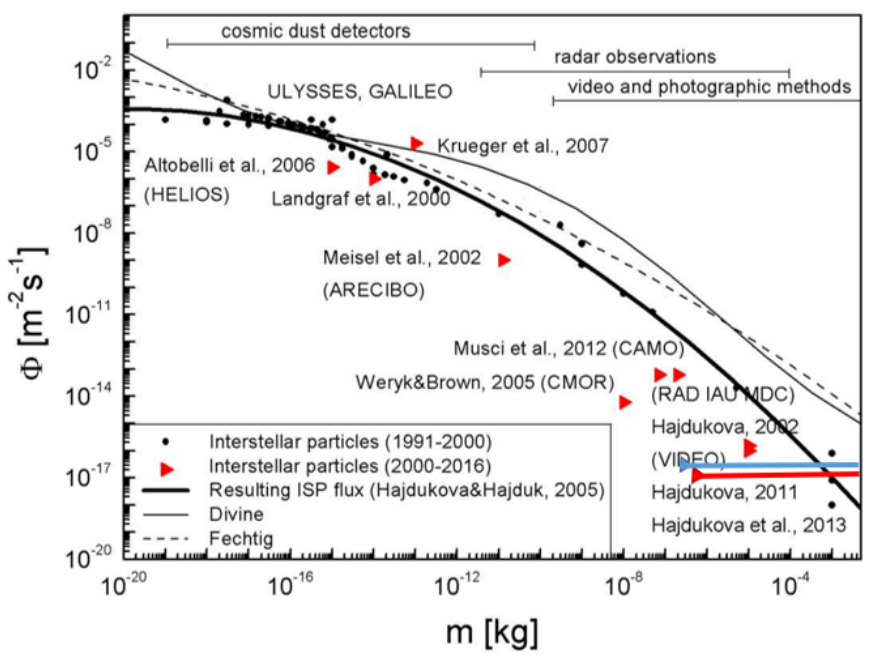

Figure 8: Expected flux limit for the observation of interstellar meteoroids using No. 1 system (red line) and No.2 system (blue line) for 1 yr observation time. meteoroids.

\section{Conclusion}

We have observed many meteors at 3 locations in Japan and USA using high sensitivity CMOS cameras to find an adequate camera system and conditions for the search for nuclearites and the observation of interstellar meteoroids in 2017 and 2018.

Observation by the DIMS (Dark matter and Interstellar Meteorid Study) experiment using 4 high sensitivity CMOS camera stations will start in a year or so at the TA site in Utah, USA. 


\section{Acknowledgement}

This work is partially supported by JSPS KAKENHI Grant Number JP19H01910 and the joint research program of the Institute for Cosmic Ray Research (ICRR), the University of Tokyo. We thanks to members of Telescope Array experiment for their help to achieve the observation, and also Appex corporation and Canon Marketing Japan Inc. for their great help related to the high sensitivity CMOS cameras.

\section{References}

[1] Edward Witten, Phys. Rev. D 30, pp. 272-285 (1984).

[2] A. De Rújula and S. L. Glashow, Nature 312, 734 (1984).

[3] Jes Madsen, Invited talk at Workshop on Exotic Physics with Neutrino Telescopes, Uppsala, Sweden, Sept. 2006, arXiv:astro-ph/0612740.

[4] David P. Anderson, Eugene T. Herrin, Vigdor L. Teplitz, Ileana M. Tibuleac, Blletine of Seismological Society of America, Vol. 107, Number 3, December 2003.

[5] Robert Hawkes, Tricia Close and Sean Woodworth, METEOROIDS 1998, Astron. Inst., Slovak Acad. Sci., Bratislava. 1999, pp. 257-264.

[6] F. Kajino, S. Takami, M. Nagasawa, M. Takahara, N. Yamamoto, M. Bertaina, A. Cellino, M. Casolino, N. Ebizuka, L.W. Piotrowski, Y. Tameda and on behalf of the JEM-EUSO Collaboration Proc. Sci. (ICRC2017) 924, 2017.

[7] SonotaCo., http://sonotaco.com.

[8] Myers J.R., Sande C.B., Miller A.C., Warren Jr. W.H., Tracewell D.A., VizieR On-line Data Catalog, Goddard Space Flight Center, Flight Dynamics Division (2002).

[9] K. A. Hill, L. A. Rogers and R. L. Hawkes, Astronomy and Astrophysics, 444, 615-624 (2005).

[10] Maria Hajdukova jr., Proceedings of the IMC, 105-110, Egmond, 2016. 